\title{
Efficacy and Toxicity of Mammalian Target Rapamycin Inhibitors in Patients with Metastatic Renal Cell Carcinoma with Renal Insufficiency: The Korean Cancer Study Group GU 14-08
}

Ki Hyang Kim, MD, PhD'
Joo Hoon Kim, MD²
Ji Young Lee, MD'
Hyo Song Kim, MD, PhD²
Su Jin Heo, MD²
Ji Hyung Kim, MD²
Ho Young Kim, MD
Sun Young Rha, MD, PhD²

${ }^{1}$ Division of Hematology-Oncology, Department of Internal Medicine, Inje University Busan Paik Hospital, Inje University College of Medicine, Busan, ${ }^{2}$ Division of Medical Oncology, Department of Internal Medicine, Yonsei Cancer Center,

Yonsei University College of Medicine, Seoul, ${ }^{3}$ Division of Hematology-Oncology, Department of Internal Medicine, Hallym University Medical Center, Hallym University College of Medicine, Seoul, Korea

\begin{abstract}
Purpose
We evaluated the efficacy and toxicity of mammalian target rapamycin inhibitors in Korean patients with metastatic renal cell carcinoma ( $\mathrm{mRCC}$ ) with chronic renal insufficiency not requiring dialysis.
\end{abstract}

\section{Materials and Methods}

Korean patients with $\mathrm{mRCC}$ and chronic renal insufficiency not requiring dialysis treated with everolimus or temsirolimus between January 2008 and December 2014 were included. Patient characteristics, clinical outcomes, and toxicities were evaluated. Overall survival (OS) and progression-free survival (PFS) durations were evaluated according to the degree of renal impairment.

\section{Results}

Eighteen patients were considered eligible for the study (median age, 59 years). The median glomerular filtration rate was $51.5 \mathrm{~mL} / \mathrm{min} / 1.73 \mathrm{~m}^{2}$. The best response was partial response in six patients and stable disease in 11 patients. The median PFS and OS durations were 8 months (95\% confidence interval [Cl], 0 to 20.4) and 32 months (95\% Cl, 27.5 to 36.5$)$, respectively. The most common non-hematologic and grade $3 / 4$ adverse events included stomatitis, fatigue, flu-like symptoms, and anorexia as well as elevated creatinine level.

\section{Conclusion}

Mammalian target rapamycin inhibitors were efficacious and did not increase toxicity in Korean patients with $\mathrm{mRCC}$ and chronic renal insufficiency not requiring dialysis.

Correspondence: Sun Young Rha, MD, PhD

Division of Medical Oncology,

Department of Internal Medicine,

Yonsei Cancer Center, Yonsei University

College of Medicine, 50-1 Yonsei-ro,

Seodaemun-gu, Seoul 03722, Korea

Tel: 82-2-2228-8130

Fax: $82-2-2227-7810$

E-mail: rha7655@yuhs.ac

Received January 14, 2016

Accepted January 28, 2016

Published Online February 12, 2016

Key words

TOR serine-threonine kinases, Renal cell carcinoma, Renal insufficiency

\section{Introduction}

With advances in the understanding of biology and genetics of metastatic renal cell carcinoma (mRCC), various tar- geted agents were developed for its treatment. These drugs targeted elements that inhibit the vascular endothelial growth factor (VEGF) and mammalian target rapamycin (mTOR) pathway [1-7].

Temsirolimus and everolimus are mTOR inhibitors used 
in treatment of mRCC. The mTOR pathway is an intracellular signaling pathway that regulates cellular metabolism, growth, proliferation, and angiogenesis [3,8]. mTOR inhibitors bind to an intracellular protein, FKBP-12, forming a complex that inhibits the mTOR serine-threonine kinase $[2,3,9]$. Temsirolimus is the standard first-line treatment for patients with poor prognosis, and everolimus is the standard second-line treatment for patients who progressed after VEGF-targeted therapy [2-4].

End-stage renal disease (ESRD) patients are at increased risk for developing cancer and at four-to-five fold increased risk of developing renal cancer in their naïve kidney $[10,11]$. Diabetes and hypertension are independent risk factors for development of renal cell carcinoma (RCC), and development of chronic kidney disease is possible in patients receiving postsurgical therapy for RCC $[12,13]$. Several studies in RCC patients with ESRD have been reported, however patients with chronic renal insufficiency not requiring dialysis have not yet been studied $[12,14,15]$. Therefore, we studied mRCC patients with chronic renal insufficiency not requiring dialysis.

The purpose of this retrospective study was to evaluate the efficacy and toxicity of mTOR inhibitors in Korean patients with $\mathrm{mRCC}$ with chronic renal insufficiency not requiring dialysis.

\section{Materials and Methods}

\section{Patients and methods}

We conducted a retrospective search for patients with $\mathrm{mRCC}$ with chronic renal insufficiency not requiring dialysis who had received the mTOR inhibitors everolimus or temsirolimus between January 2008 and December 2014 at Yonsei Cancer Center and Busan Paik Hospital, in South Korea. The Cockcroft-Gault formula was used for calculation of the glomerular filtration rate (GFR). Patients with a GFR of $\geq 15$ $\mathrm{mL} / \mathrm{min} / 1.73 \mathrm{~m}^{2}$ but $<60 \mathrm{~mL} / \mathrm{min} / 1.73 \mathrm{~m}^{2}$ were considered eligible for analysis. The patients were divided into two groups according to the degree of renal insufficiency, as defined by the National Kidney Foundation [24]: moderate renal impairment $\left(30 \mathrm{~mL} / \mathrm{min} / 1.73 \mathrm{~m}^{2} \leq \mathrm{GFR}<60 \mathrm{~mL} / \mathrm{min} /\right.$ $\left.1.73 \mathrm{~m}^{2}\right)$ and severe renal impairment $\left(15 \mathrm{~mL} / \mathrm{min} / 1.73 \mathrm{~m}^{2}\right.$ $\leq \mathrm{GFR}<30 \mathrm{~mL} / \mathrm{min} / 1.73 \mathrm{~m}^{2}$ ). The following clinical data were obtained retrospectively: demographics (age and sex), Eastern Cooperative Oncology Group (ECOG) performance status, stage at diagnosis, prognostic risk group based on the Memorial Sloane Kettering Cancer Center Criteria (MSKCC), results after prior nephrectomy, and serum creatinine con- centrations. The following data regarding mTOR inhibitors were obtained: initial dose and schedule of mTOR inhibitors, serum creatinine concentration during and after use of mTOR inhibitors, dose reductions, and adverse events (AEs) and abnormal laboratory findings graded according to the National Cancer Institute Common Terminology Criteria for AEs ver. 3.0. The best response defined according the Response Evaluation Criteria In Solid Tumors (RECIST), progression-free survival (PFS), and overall survival (OS) data were also collected. PFS was defined as time from date of first dose of mTOR inhibitors to the first documentation of disease progression or death from any cause; OS was defined as time from date of first dose of mTOR inhibitors to the final documentation of death from any cause or to last follow-up. The study was approved by the Protocol Review Committee of the Korean Cancer Study Group (KCSG GU) 14-08.

\section{Statistical analysis}

Categorical data are presented as frequency counts and percentages, and continuous variables, as medians and ranges. PFS and OS durations were evaluated using the Kaplan-Meier method. Log-rank tests were used for comparison of PFS and OS data between the two patient groups and by mTOR inhibitor regimen (everolimus or temsirolimus). All analyses were performed using SPSS ver. 22.0 (IBM Co., Armonk, NY).

\section{Results}

\section{Patient characteristics}

From both centers, 18 patients were eligible. Patient characteristics are listed in Table 1. The median age at diagnosis was 59 years. Fifteen patients had clear cell histology and three patients had non-clear cell histology. All patients had renal insufficiency at diagnosis of RCC. Eight of these patients had renal insufficiency due to hypertension and diabetes, but others had renal insufficiency due to unknown causes. Sixteen patients had undergone prior nephrectomy. The median GFR was $51.2 \mathrm{~mL} / \mathrm{min} / 1.73 \mathrm{~m}^{2}$. Sixteen patients were classified as having moderate renal insufficiency and two as having severe renal insufficiency. In the moderate renal insufficiency group, five patients were treated with mTOR inhibitors as a first-line treatment. Two patients in this group were treated with bevacizumab plus temsirolimus due to enrollment in a clinical trial, and two were treated with temsirolimus due to non-clear cell histology. One patient in this group was treated with everolimus due to enrollment in 
Table 1. Characteristics of mRCC patients with renal insufficiency

\begin{tabular}{|c|c|c|c|}
\hline Characteristic & Moderate renal impairment & Severe renal impairment & Total \\
\hline \multicolumn{4}{|l|}{ Sex } \\
\hline Male & 14 & 1 & 15 \\
\hline Female & 2 & 1 & 3 \\
\hline Age at diagnosis, median (range, yr) & $56(44-81)$ & $73.5(73-74)$ & 59 \\
\hline \multicolumn{4}{|l|}{ ECOG } \\
\hline 0 & 9 & 0 & 9 \\
\hline 1 & 7 & 2 & 9 \\
\hline \multicolumn{4}{|l|}{ Histology } \\
\hline Clear-cell & 13 & 2 & 15 \\
\hline Non-clear cell & 3 & 0 & 3 \\
\hline \multicolumn{4}{|l|}{ Stage at diagnosis } \\
\hline I & 1 & 0 & 1 \\
\hline II & 3 & 0 & 3 \\
\hline III & 0 & 0 & 0 \\
\hline IV & 10 & 2 & 12 \\
\hline Unknown & 2 & 0 & 2 \\
\hline Prior nephrectomy & 14 & 2 & 16 \\
\hline \multicolumn{4}{|l|}{ Underlying disease } \\
\hline Hypertension and diabetes & 3 & 1 & \\
\hline Diabetes & 3 & 0 & \\
\hline Hypertension & 1 & 0 & \\
\hline $\begin{array}{l}\text { Ccr level at start date of } \mathrm{mTOR} \text { inhibitors, } \\
\text { median (range, } \mathrm{mL} / \mathrm{min} / 1.73 \mathrm{~m}^{2} \text { ) }\end{array}$ & $51.9(35-59.7)$ & $22(18-26)$ & $51.2(18-59.7)$ \\
\hline \multicolumn{4}{|l|}{ MSKCC risk groups } \\
\hline Favorable & 6 & 1 & 7 \\
\hline Intermediate & 9 & 1 & 10 \\
\hline Poor & 1 & 0 & 1 \\
\hline \multicolumn{4}{|l|}{ No. of prior regimens } \\
\hline 0 & 5 & 0 & 5 \\
\hline 1 & 11 & 2 & 13 \\
\hline
\end{tabular}

mRCC, metastatic renal cell carcinoma; ECOG, Eastern Cooperative Oncology Group; Ccr, creatinine clearance; mTOR, mammalian target rapamycin; MSKCC, Memorial Sloane Kettering Cancer Center Criteria.

a clinical trial. Eleven patients were treated with mTOR inhibitors as a second-line treatment, including four who were treated with temsirolimus and the others with everolimus. All patients with severe renal impairment had an ECOG performance status of 1 and had undergone prior nephrectomy, although all patients presented with stage IV disease at diagnosis.

All patients with severe renal impairment were treated with everolimus as a second-line treatment. One patient was treated with sunitnib as a first-line treatment, and another with sorafenib. In the severe renal impairment group, the median GFR of patients was $22 \mathrm{~mL} / \mathrm{min} / 1.73 \mathrm{~m}^{2}$. According to MSKCC risk grouping, one patient with severe renal impairment had a favorable prognosis and one patient had an intermediate prognosis.

\section{Safety assessment of mTOR inhibitor treatment}

The starting oral dose of everolimus was $10 \mathrm{mg}$ daily for 10 patients and the starting intravenous dose for temsirolimus was $25 \mathrm{mg}$ weekly for eight patients in the overall series. All patients were started on the original dose of mTOR inhibitors regardless of GFR. In the moderate renal impairment group, six patients treated with everolimus were dose delayed due to non-renal toxicity, including one patient treated with everolimus as a first-line treatment and the others treated with everolimus as a second-line treatment. Three patients treated with temsirolimus were dose delayed due to non-renal toxicity, including one patient treated with temsirolimus as a first-line treatment and two patients treated with temsirolimus as a second-line treatment. 
Table 2. Adverse events of all patients included in the study

\begin{tabular}{|c|c|c|c|}
\hline Adverse event & Grade 1-2 & Grade 3-4 & All grades \\
\hline Fatigue & $3(16)$ & 0 & $3(16)$ \\
\hline General pain & $1(5)$ & 0 & $1(5)$ \\
\hline Skin color change & $1(5)$ & 0 & $1(5)$ \\
\hline Flu-like symptoms & $3(16)$ & 0 & $3(16)$ \\
\hline Taste alteration & $1(5)$ & 0 & $1(5)$ \\
\hline Pruritus & $1(5)$ & 0 & $1(5)$ \\
\hline General edema & $1(5)$ & 0 & $1(5)$ \\
\hline Stomatitis & $9(50)$ & 0 & $9(50)$ \\
\hline Anorexia/Nausea & $3(16)$ & 0 & $3(16)$ \\
\hline Dyspepsia & $2(11)$ & 0 & $2(11)$ \\
\hline Cough & $2(11)$ & 0 & $2(11)$ \\
\hline \multicolumn{4}{|l|}{ Laboratory abnormality } \\
\hline Anemia & $1(5)$ & 0 & $1(5)$ \\
\hline Creatinine level elevation & $13(72)$ & $1(5)$ & $14(77)$ \\
\hline Hypertriglyceridemia & $1(5)$ & 0 & $1(5)$ \\
\hline Hyperglycemia & $2(11)$ & 0 & $2(11)$ \\
\hline
\end{tabular}

Values are presented as number $(\%)$.

Table 3. Changes of median GFR of patients according to the degree of renal insufficiency

\begin{tabular}{lccc} 
Variable & $\begin{array}{c}\text { Before mTOR } \\
\text { inhibitors treatment }\end{array}$ & $\begin{array}{c}\text { During mTOR } \\
\text { inhibitors treatment }\end{array}$ & $\begin{array}{c}\text { After mTOR } \\
\text { inhibitors treatment }\end{array}$ \\
Moderate renal impairment & $51.9(35-59.7)$ & $36.3(11-58.1)$ & $49(8.9-74)$ \\
Severe renal impairment & $22(18-26)$ & $17(15-19)$ & $25(25)$ \\
\hline
\end{tabular}

Values are presented as median (range). GFR, glomerular filtration rate; mTOR, mammalian target rapamycin.

In the moderate renal insufficiency group, six patients had dose reduction because of non-renal toxicity. Four of them received everolimus as a second-line treatment, and two received temsirolimus as a second-line treatment. The median duration of delay was 2 weeks for everolimus and 2 weeks for temsirolimus. In the moderate renal insufficiency group, one patient had dose delay and dose reduction because of grade 3 creatinine level elevation; this patient was receiving bevacizumab plus temsirolimus as a first-line treatment. None of the patients with severe renal insufficiency experienced delays or required dose reductions. The median relative dose intensity during mTOR inhibitor treatment was 97\% for the everolimus group and $98 \%$ for the temsirolimus group. The treatment-related AEs are summarized in Table 2 . The most common non-hematologic and grade $3 / 4$ AEs included stomatitis, fatigue, flu-like symptoms, and anorexia as well as creatinine level elevation. The changes of median GFR of patients according to the degree of renal insufficiency are summarized in Table 3.

\section{Treatment outcomes}

Of the 18 patients, three achieved partial response, the best tumor response observed, of whom two received bevacizumab plus temsirolimus as a first-line treatment and one received temsirolimus as a second-line treatment; 14 patients showed stable disease. Overall response rate (ORR) was $16 \%$ and disease-control rate was $94 \%$. The median PFS duration was 8 months (95\% confidence interval [CI], 0 to 20.5) and the median OS duration was 25 months (95\% CI, 12.9 to 37.1). In patients treated with mTOR inhibitors as a first-line treatment, median PFS duration was 32 months (95\% CI, not estimated) and the median OS duration was 32 months (95\% $\mathrm{CI}, 8.7$ to 55.2). In patients treated with mTOR inhibitors as a second-line treatment, median PFS duration was 4 months (95\% CI, 1.1 to 6.9) and the median OS duration was 18 months (95\% CI, 6.9 to 29.1) (Fig. 1). We evaluated PFS and OS rates in patients stratified according to the severity of renal impairment. In the group with moderate/stage 3 renal 
A

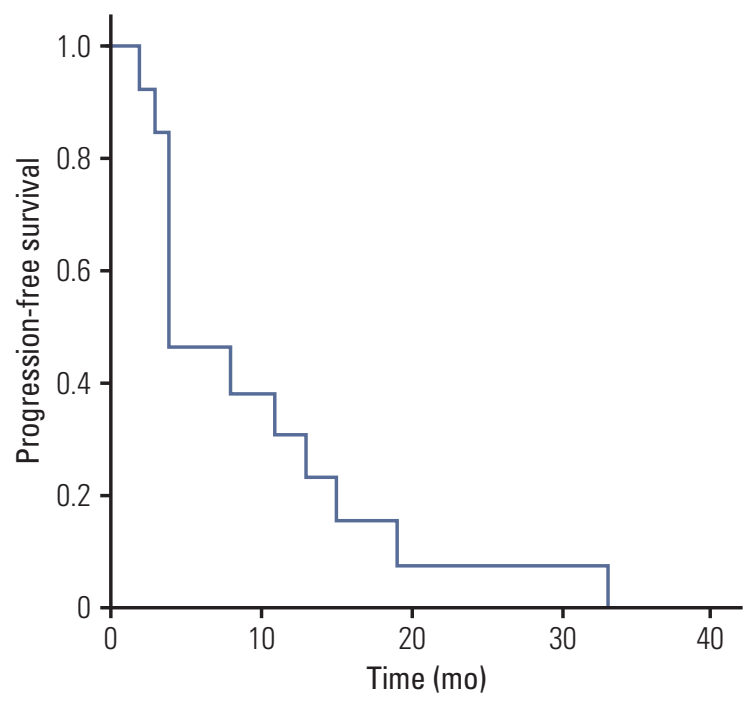

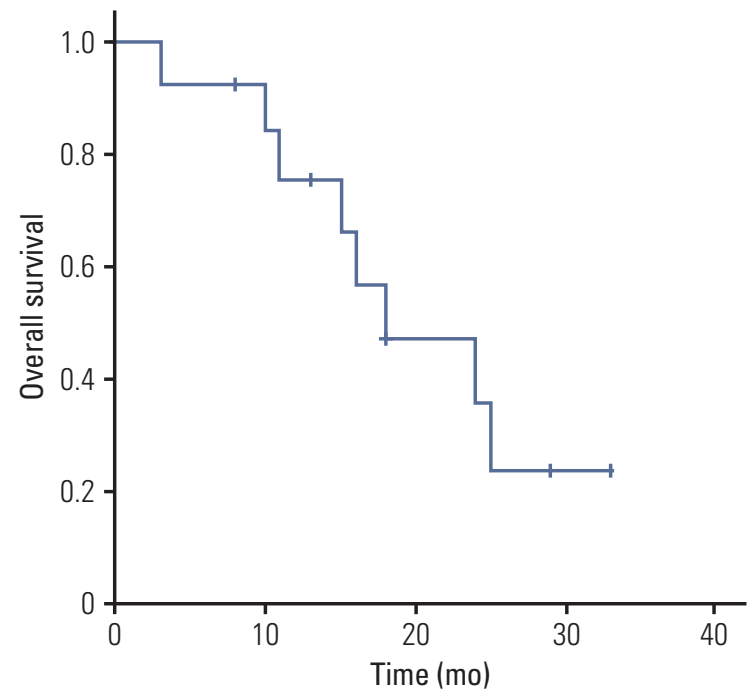

Fig. 1. Kaplan-Meier estimates of progression-free survival (A) and overall survival (B) durations in patients treated with mammalian target rapamycin inhibitors as a second-line treatment $(n=18)$. (A) Median progression-free survival: 4 months (95\% confidence interval [CI], 1.1 to 6.9). (B) Median overall survival: 18 months (95\% CI, 6.9 to 29.1).

impairment, the median PFS duration was 5.0 months $(95 \%$ CI, 0 to 12.8) and the median OS duration was 25 months (95\% CI, 13.9 to 36.1 ).

We also evaluated PFS and OS rates in patients stratified according to the mTOR inhibitor regimens. Patients treated with temsirolimus and those treated with temsirolimus plus bevacizumab were included in the analysis. In patients treated with temsirolimus, the median PFS duration was 13 months (95\% CI, 0 to 33.8) and the median OS duration was 18 months (95\% CI, 0 to 38.8). In patients treated with everolimus, the median PFS duration was 5 months $(95 \% \mathrm{CI}$, 0 to 11.2) and the median OS was 24 months ( $95 \%$ CI, 9.2 to 38.8).

\section{Discussion}

In this retrospective study, patients with $\mathrm{mRCC}$ and renal insufficiency treated with mTOR inhibitors showed median PFS and OS durations of 8 and 25 months, respectively. The ORR was $16 \%$ and the disease-control rate was $94 \%$. Our study thus yielded mixed data for mTOR inhibitor regimens. Of 18 patients, five patients received mTOR inhibitors as a first-line treatment and 13 as a second-line treatment. Eight patients received temsirolimus, including bevacizumab plus temsirolimus, and 10 patients received everolimus. Therefore, it is difficult to compare the current phase III data with those of other studies. However, we compared data with those of three studies: a phase III study on everolimus in advanced renal cell carcinoma with normal renal function that had progressed on other target therapies; one on temsirolimus used as a first-line treatment; and one on temsirolimus used as a second-line therapy after sunitinib [2-4]. In the results of previous phase III studies on mRCC patients with normal renal function, median PFS was 4 months for everolimus, 5.5 months for temsirolimus, and 4.3 months for temsirolimus. The median OS with everolimus was not determined, 10.9 months for temsirolimus and 12.3 months for temsirolimus [2-4]. In our study, both median PFS and OS durations of $\mathrm{mRCC}$ patients with impaired renal function were longer than those in $\mathrm{mRCC}$ patients with normal renal function. The ORR in previous phase III studies with everolimus and temsirolimus were $1 \%$ and $8 \%-8.6 \%$, respectively, lower than those in our study. The AEs of mTOR inhibitors in patients with renal insufficiency were similar to those in mRCC patients with normal renal function treated with mTOR inhibitors in previous phase III studies [2-4]. Therefore, mTOR inhibitors had the same efficacy in patients with renal insufficiency, although this study was a retrospective study.

Because both everolimus and temsirolimus are cleared by the liver and are mainly excreted in feces, all patients in our 
study were started on the original dose regimen of everolimus and temsirolimus [16-18]. Only one patient experienced a delay in dose and dose reduction due to grade 3 creatinine level elevation during treatment. Dose intensity of patients in our study was 97\%-98\%. Therefore, mTOR inhibitors did not increase renal toxicity or non-renal toxicity in mRCC patients with renal insufficiency.

Everolimus and temsirolimus are derivatives of sirolimus, which is used in treatment of renal transplants; both drugs are cleared by the liver and are mainly excreted in feces, therefore we can overlook that mTOR inhibitors are safe for patients with renal insufficiency. However, according to previous phase III studies and our study, the incidence of creatinine level elevation in patients with normal renal function or renal insufficiency treated with mTOR inhibitors was approximately 50\% [4,8]. Also, renal function of mRCC patients may have deteriorated because of aging, chronic co-morbidities, and previous partial or radical nephrectomy $[12,13,19]$. Therefore, careful monitoring of kidney function is required in mRCC patients taking mTOR inhibitors.

However, although our study was a small retrospective study that used heterogeneous treatments of mTOR inhibitors, our results showed that the use of mTOR inhibitors in patients with $\mathrm{mRCC}$ with renal insufficiency not requiring dialysis was efficacious and did not increase toxicities.

\section{Conclusion}

mTOR inhibitors were found to be efficacious and safe for the treatment of mRCC patients with renal insufficiency not requiring dialysis, however the renal function of these patients should be monitored during treatment.

\section{Conflicts of Interest}

Conflict of interest relevant to this article was not reported.

\section{Acknowledgments}

This study was supported by a grant from the National R\&D Program for Cancer Control, Ministry of Health and Welfare, Republic of Korea (1520190). The research was supported by the Korean Cancer Study Group.

\section{References}

1. Rini BI, Halabi S, Rosenberg JE, Stadler WM, Vaena DA, Ou SS, et al. Bevacizumab plus interferon alfa compared with interferon alfa monotherapy in patients with metastatic renal cell carcinoma: CALGB 90206. J Clin Oncol. 2008;26:5422-8.

2. Motzer RJ, Escudier B, Oudard S, Hutson TE, Porta C, Bracarda $S$, et al. Efficacy of everolimus in advanced renal cell carcinoma: a double-blind, randomised, placebo-controlled phase III trial. Lancet. 2008;372:449-56.

3. Hudes G, Carducci M, Tomczak P, Dutcher J, Figlin R, Kapoor A, et al. Temsirolimus, interferon alfa, or both for advanced renal-cell carcinoma. N Engl J Med. 2007;356:2271-81.

4. Hutson TE, Escudier B, Esteban E, Bjarnason GA, Lim HY, Pittman KB, et al. Randomized phase III trial of temsirolimus versus sorafenib as second-line therapy after sunitinib in patients with metastatic renal cell carcinoma. J Clin Oncol. 2014;32:760-7.

5. Sternberg CN, Davis ID, Mardiak J, Szczylik C, Lee E, Wagstaff J, et al. Pazopanib in locally advanced or metastatic renal cell carcinoma: results of a randomized phase III trial. J Clin Oncol. 2010;28:1061-8.

6. Escudier B, Eisen T, Stadler WM, Szczylik C, Oudard S, Siebels $\mathrm{M}$, et al. Sorafenib in advanced clear-cell renal-cell carcinoma. N Engl J Med. 2007;356:125-34.
7. Motzer RJ, Hutson TE, Tomczak P, Michaelson MD, Bukowski RM, Oudard S, et al. Overall survival and updated results for sunitinib compared with interferon alfa in patients with metastatic renal cell carcinoma. J Clin Oncol. 2009;27:3584-90.

8. Oudard S, Medioni J, Aylllon J, Barrascourt E, Elaidi RT, Balcaceres J, et al. Everolimus (RAD001): an mTOR inhibitor for the treatment of metastatic renal cell carcinoma. Expert Rev Anticancer Ther. 2009;9:705-17.

9. Iacovelli R, Palazzo A, Trenta P, Mezi S, Pellegrino D, Naso G, et al. Management of metastatic renal cell carcinoma progressed after sunitinib or another antiangiogenic treatment. Am J Clin Oncol. 2014;37:611-5.

10. Woldu SL, Weinberg AC, RoyChoudhury A, Chase H, Kalloo SD, McKiernan JM, et al. Renal insufficiency is associated with an increased risk of papillary renal cell carcinoma histology. Int Urol Nephrol. 2014;46:2127-32.

11. Hofmann JN, Purdue MP. CKD and risk of renal cell carcinoma: a causal association? J Am Soc Nephrol. 2014;25: 2147-8.

12. Russo P. End stage and chronic kidney disease: associations with renal cancer. Front Oncol. 2012;2:28.

13. Chang A, Finelli A, Berns JS, Rosner M. Chronic kidney disease in patients with renal cell carcinoma. Adv Chronic Kid- 
ney Dis. 2014;21:91-5.

14. Shetty AV, Matrana MR, Atkinson BJ, Flaherty AL, Jonasch E, Tannir NM. Outcomes of patients with metastatic renal cell carcinoma and end-stage renal disease receiving dialysis and targeted therapies: a single institution experience. Clin Genitourin Cancer. 2014;12:348-53.

15. Neuzillet Y, Tillou X, Mathieu R, Long JA, Gigante M, Paparel $\mathrm{P}$, et al. Renal cell carcinoma ( $R C C$ ) in patients with end-stage renal disease exhibits many favourable clinical, pathologic, and outcome features compared with RCC in the general population. Eur Urol. 2011;60:366-73.

16. Letavernier E, Legendre C. mToR inhibitors-induced protein- uria: mechanisms, significance, and management. Transplant Rev (Orlando). 2008;22:125-30.

17. Simpson D, Curran MP. Temsirolimus: in advanced renal cell carcinoma. Drugs. 2008;68:631-8.

18. Kovarik JM, Sabia HD, Figueiredo J, Zimmermann $H$, Reynolds C, Dilzer SC, et al. Influence of hepatic impairment on everolimus pharmacokinetics: implications for dose adjustment. Clin Pharmacol Ther. 2001;70:425-30.

19. Kim KH, Kim HY, Kim HR, Sun JM, Lim HY, Lee HJ, et al. Efficacy and toxicity of sunitinib in patients with metastatic renal cell carcinoma with renal insufficiency. Eur J Cancer. 2014;50:746-52. 